\title{
Respeto y tolerancia como valores promotores de la Cultura de Paz en padres de niños en edad preescolar en el municipio de San Nicolás de los Garza, Nuevo León, México* \\ Respect and tolerance as promoting values to the Cullture of Peace in parents of pre-school children in the city of San Nicolás de los Garza, Nuevo León, Mexico Respeito e tolerância como valores promocionais da Cultura de Paz em pais de crianças em idade pré-escolar no município de San Nicolas de los Garza, Nuevo Leon, México
}

\author{
Karla Rodríguez-Burgos ${ }^{\star *}$ \\ (iD http://orcid.org/0000-0003-2093-8146 \\ Myrna Hinojosa García ${ }^{\star * *}$ \\ (iD $h$ ttp://orcid.org/0000-0002-9149-7774 \\ Universidad Autónoma de Nuevo León, México
}

\begin{abstract}
Resumen
La siguiente investigación pertenece al proyecto Beneficios de la Cultura de Paz aplicada a las relaciones paterno filiales, de la Facultad de Derecho y Criminología de la Universidad Autónoma de Nuevo León, auspiciado por el Concejo Nacional de Ciencia y Tecnología (CONACYT). En este artículo se presentan los resultados de un preexperimento aplicado a una muestra de padres de familia con hijos en edad preescolar, los cuales participaron de manera voluntaria en un curso para formarlos como capacitadores de sus hijos en la Cultura de Paz en el municipio de San Nicolás de los Garza, Nuevo León, México. Debido a que el respeto y la tolerancia son valores fundamentales para la convivencia pacífica, la presente investigación se centra en los resultados obtenidos respecto al aumento o disminución de manifestaciones de dichos valores en los participantes del curso.

Palabras clave: Respeto, Tolerancia, Cultura de Paz, Padres de familia.
\end{abstract}

Abstract

This research belongs to the following Project "Beneficios de la Cultura de Paz aplicada a las relaciones paterno filiales", from the Facultad de Derecho y Criminología de la Universidad Autónoma de Nuevo León, supported by Concejo Nacional de Ciencia y Tecnología (CONACYT). On this paper is presented the results of a first experience applied to a sample of parents whose children are in preschool age, those parents were volunteers in a course with the objective to make them trainers for a Peace Culture in the city of San Nicolás de los Garza, Nuevo León, México. Respect and tolerance are fundamental values for a peaceful living, so this research is centered on the results upon the increase or decrease of those values manifestation between the volunteers on the course.

Keywords: Respect, Tolerance, Peace Culture, Parents.

Resumo

O seguinte projeto de pesquisa pertence ao projeto Benefícios da Cultura de Paz aplicado às relações paterno-filiais, de Faculdade de Direito e Criminologia na Universidade Autônoma de Nuevo Leon, patrocinado pelo Conselho Nacional Ciência e Tecnologia (CONACYT). Este artigo descreve os resultados de uma experiência aplicada a uma amostra de pais de família com crianças em idade pré-escolar, que participaram voluntariamente em um curso de treinamento como capacitadores para seus filhos na Cultura de Paz no município de San Nicolas de los Garza, Nuevo Leon, México. Devido a que o respeito e a tolerância são valores fundamentais para a convivência pacífica, esta pesquisa se concentra em resultados referentes ao aumento ou diminuição na manifestação destes valores nos participantes do curso.

Palavras-chave: Respeito, Tolerância, Cultura de paz, Pais de família.

Cómo referenciar este artículo: Rodríguez, K. \& Hinojosa, M. (2017). Respeto y tolerancia como valores promotores de la Cultura de Paz en padres de niños en edad preescolar en el municipio de San Nicolás de los Garza, Nuevo León, México. Pensamiento Americano, 10(18), 96-114.

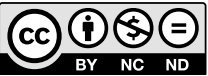

Recibido: Junio 5 de 2016 • Aceptado: Septiembre 27 de 2016

* Beneficios de la Cultura de Paz aplicada a las relaciones paterno-filiales, financiado por Conacyt y la Universidad Autónoma de Nuevo León, México.

** Doctora en Ciencias Políticas. Coordinadora del Doctorado en Filosofía con orientación en Ciencias Políticas, Universidad Autónoma de Nuevo León, México.

*** Candidata a Doctor en Métodos Alternos de Solución de Conflictos, Universidad Autónoma de Nuevo León, México. 


\section{Introducción}

Reconociendo la importancia de los elementos y valores fundamentales de la Cultura de Paz, como lo son el respeto y la tolerancia, el presente artículo se centra en analizar e interpretar los resultados de un preexperimento aplicado a una muestra de padres de familia con hijos en edad preescolar, habitantes del municipio de San Nicolás de los Garza, Nuevo León, México.

Con el objetivo de formar a los padres como capacitadores de sus hijos en la Cultura de Paz se diseñó e implementó un programa cuyo contenido teórico se conforma de los elementos de la Cultura de Paz. La Organización de las Naciones Unidas, en el Artículo 1 de la Resolución A/RES/53/243 (ONU, 1999) refiere que la Cultura de Paz consiste en un conjunto de valores, actitudes, tradiciones, comportamientos y estilos de vida basados en:

a) El respeto a la vida, el fin de la violencia y la promoción y práctica de la no violencia por medio de la educación, el diálogo y la cooperación.

b) El respeto pleno de los principios de soberanía, integridad territorial e independencia política de los Estados y de no injerencia en los asuntos que son esencialmente jurisdicción interna de los Estados, de conformidad con la Carta de las Naciones Unidas y el Derecho Internacional.

c) El respeto pleno y la promoción de todos los derechos humanos y las libertades fundamentales.

d) El compromiso con el arreglo pacífico de los conflictos. e) Los esfuerzos para satisfacer las necesidades de desarrollo y protección del medioambiente de las generaciones presentes y futuras.

f) El respeto y la promoción del derecho al desarrollo.

g) El respeto y el fomento de la igualdad de derechos y oportunidades de mujeres y hombres.

h) El respeto y el fomento del derecho de todas las personas a la libertad de expresión, opinión e información.

i) La adhesión a los principios de libertad, justicia, democracia, tolerancia, solidaridad, cooperación, pluralismo, diversidad cultural, diálogo y entendimiento a todos los niveles de la sociedad y entre las naciones; $y$ animados por un entorno nacional e internacional que favorezca a la paz.

El programa diseñado tuvo una duración de seis sesiones en las cuales se trabajó con los padres de familia para adquirir conocimientos acerca de la paz, la violencia, el conflicto y reforzar estrategias y herramientas de disciplina y solución de conflictos basadas en el respeto. Se generaron las condiciones para la reflexión acerca de la importancia de retomar el respeto hacia uno mismo como base y forma de diálogo interno.

\section{Respeto y tolerancia como valores promo- tores de una convivencia pacífica}

El respeto es esencial en las relaciones, tanto intrapersonales como interpersonales, dentro de un grupo y de una sociedad. Es la madre 
de todas las virtudes (von Hildebrand, 2004), ya que constituye la actitud fundamental que presuponen todas ellas. En el respeto se conforma el propio criterio al valor fundamental de lo existente, se le reconoce y se le da a lo existente la oportunidad de desplegarse, de que comparta su experiencia.

Consiste en tener una actitud abierta y tolerante hacia las opiniones, creencias y formas de expresión de otros con el objetivo de lograr la convivencia en armonía. Es el reconocimiento de que algo o alguien tienen valor y dignidad, a pesar de ser diferente; consiste en que una persona o un grupo reconozca, considere y aprecie las cualidad des de algo propio o ajeno (Modelo de Desarrollo Económico de Navarra, 2010).

Es un elemento constitutivo del asombro, la curiosidad de conocer lo que es diferente o desconocido, sin generar un rechazo previo, sino con una actitud de apertura. El respeto permite apreciar al otro y a uno mismo, conocer, reconocer las diferencias y similitudes, proporciona las bases para relacionarse de una forma constructiva, así mismo propicia el crecimiento personal por medio del aprendizaje. Respetar es indispensable para comprender y vivir los demás valores.

Respetarse a uno mismo implica observar la propia realidad, así como aceptar la identidad y dignidad, con una actitud abierta, de aceptación y tolerante hacia las propias creencias y formas de expresión sin rechazarlas o declararlas como las única con validez, no es ni un extremo ni el otro (Modelo de Desarrollo Económico de Navarra, 2010). La presencia del autorrespeto se manifiesta en la capacidad de aceptación y validación que se tiene de las experiencias de otras personas. Quien no se respeta a sí mismo no manifestará respeto hacia los demás, partiendo desde el principio de que no se puede dar lo que no se tiene.

Para la convivencia pacífica el respeto debe ser bilateral, otorgarse al mismo tiempo que se solicita. En las interacciones interpersonales para ser respetado se requiere respetar, ya que en los procesos de comunicación las personas tienden a responder dependiendo de la forma en la que se transmita el mensaje: si el emisor expresa el mensaje de forma respetuosa, y el contenido del mismo sigue los mismos parámetros, el receptor tiende a responder de la misma forma (Pérez, 2009).

Aplicando el mismo razonamiento, en el proceso de enseñanza-aprendizaje de padres hacia los hijos, para que los niños aprendan e internalizan el respeto es necesario demostrárselos en el trato y convivencia. Mostrar aceptación a los derechos y obligaciones de los demás fomenta el recibir el mismo trato de parte de ellos. Respetar es reconocer que una cosa, persona o situación tiene valor, por lo que debe ser apreciado, apreciadas las diferencias y significado (Modelo de Desarrollo Económico de Navarra, 2010).

El respeto como creencia se refleja en actitudes de equidad, igualdad, amabilidad, dis- 
posición a la escucha, empatía y curiosidad. La curiosidad es una forma de validar la experiencia del otro, un deseo de conocer y explorar esta experiencia implica que se reconocen las diferencias entre la experiencia personal y la del otro, existiendo una disposición de conocer y de aprender.

La falta de respeto a un individuo (von Hildebrand, 2004) se manifiesta en actitudes de superioridad, insolencia, presunción, en tratos superficiales, sin comprensión de la situación. El individuo que no se respeta ni se reconoce a sí mismo, tiene una necesidad de aprobarse hacia el exterior, de ser aceptado; busca satisfacer esta necesidad con desesperación, lo cual le impide detectar a las personas, cosas o situaciones en su entorno, solamente busca su propia validación.

Según el niño sea tratado es como aprenderá a tratarse a sí mismo y a los demás. Cuando no se respetan las necesidades, opinión y presencia, el menor desarrolla una necesidad de validación, busca llamar la atención ayudándose de cualquier método que le sea útil, siendo de los más comunes la mala conducta. Sin tener un antecedente de respeto, el niño difícilmente podrá procurar su propio bienestar y el de los demás. El respeto es fundamental para el desarrollo de la empatía, la capacidad de ponerse en el lugar de otras personas, así como de la tolerancia, valores básicos para la convivencia.

Para poder desarrollar la tolerancia es nece- sario tener la capacidad de contemplar las divergencias de los demás con respeto, dándole a las personas la oportunidad de ser diferentes y el derecho de poder coexistir en el mismo espacio; una vez que se puede respetar a los demás, observando las diferencias con curiosidad y respeto, es posible reconocer y elegir una postura al respecto, de acuerdo o en desacuerdo. En caso de que la postura sea la contraria, la tolerancia es la capacidad de poder respetar a otras personas aunque sus ideas difieran de las propias.

La tolerancia consiste en el respeto, aceptación así como aprecio de la rica diversidad de las culturas, creencias, tradiciones y costumbres, de las formas de expresión y medios de ser humanos. Es así que la tolerancia es fomentada por el conocimiento, la actitud de apertura, la comunicación y la libertad de pensamiento, de conciencia y de religión. Consiste en la armonía en la diferencia, es una virtud que hace posible la paz y contribuye al cambio de paradigmas: de la violencia cultural hacia la cultura de paz (UNESCO, 1995).

Aplicada en las relaciones con uno mismo mediante la aceptación de las características personales, preferencias y gustos, la falta de tolerancia en las relaciones intrapersonales se manifiesta en un autojuicio punitivo o destructivo, en una falta de autoconfianza y autoestima. La falta de tolerancia en las relaciones interpersonales se expresa mediante conductas que dan preferencia a las propias creencias sobre las de los demás, e impone a otros la propia voluntad sin considerar sus necesidades. 
La tolerancia es necesaria entre los individuos y dentro de los grupos sociales, para lograr la enseñanza-aprendizaje es necesario inculcar en las instituciones formadoras, como la familia, escuela y sociedad, actitudes de apertura, solidaridad y comunicación, como es el caso de la escucha activa. El aprendizaje por imitación es la principal herramienta de adquisición de nuevas conductas, para aprovecharlo en la transmisión de la tolerancia los padres deben conocer en qué consiste, analizar sus creencias, actitudes y comportamientos e identificar si reflejan la tolerancia.

La educación es el medio más eficaz para prevenir la intolerancia (UNESCO, 1995). Se inicia enseñando a las personas los derechos y libertades que comparten, para que puedan ser reconocidos, aceptados, respetados, para fomentar la voluntad de proteger los derechos propios así como los de los demás. Es necesario realizar un análisis para conocer las raíces de la intolerancia y la exclusión, estudiarlas para clarificar que su base es la falta de respeto, el temor y la ignorancia. De ahí surge la importancia de reforzar en la educación familiar los valores de respeto y tolerancia, labor para la cual los padres son agentes de cambio idóneos, enseñando mediante el ejemplo conductas basadas en el respeto y la tolerancia.

\section{Los padres como agentes de paz}

El proceso de desarrollo y aprendizaje tiene especial importancia en la infancia ya que los niños comienzan a comportarse y funcionar como miembros de la sociedad. Los agentes de socialización como la familia, los padres, la escuela y los medios de comunicación cumplen una función preponderante en la formación integral de una persona (Cárdenas-Flores, Cosiatado-Carrasco \& Livia-Vicente, 2011).

La familia es el principal agente socializador del individuo, el niño adquiere los primeros contactos con el ambiente a través de sus padres y familia, desarrollando de esta manera conductas adaptativas que pueden ser positivas o negativas, dependiendo si las primeras experiencias son gratificantes o no (Flores Millán, 2011). Por lo que, es importante reforzar en el seno familiar valores como el respeto, la tolerancia y solidaridad, los cuales son transmitidos por los padres hacia los hijos.

El comportamiento de los padres es un modelo a seguir para el niño, ya que les enseña acerca de la disciplina, la actitud y la conducta, que son pilares del carácter del individuo. Estos aspectos son factores muy influyentes en el aprendizaje de la conducta agresiva. Flores Millán (2011) refiere que el tipo de disciplina que los padres aplican al niño, tiene relación con el tipo de conductas que el menor utiliza para relacionarse con él mismo y con los demás. La imitación es el mecanismo de aprendizaje más frecuente en los niños y sienta las bases del aprendizaje futuro (Bajo, Campillo, Pérez, Gener, Gallego \& Padrino, 2011).

Debido a su potencial como agente formador se han desarrollado intervenciones para el trabajo directo con los padres, cuyos objetivos 
son diversos. La mayoría de estos programas están dirigidos a la disminución y erradicación de la violencia infantil (Cornella i Canals \& Llusent i Guillament, 2010). La familia como plataforma de transmisión de la Cultura de Paz ha sido objeto de estudio y objetivo de diversos de programas internacionales, los cuales se han desarrollado y llevado a cabo por parte de la Organización de las Naciones Unidas para la Educación, la Ciencia y la Cultura (UNESCO).

Se diseñó e impartió un programa cuyo objetivo fue que los padres obtuvieran información, conceptos aplicados y recursos que les permitieran ampliar su conocimiento $\mathrm{y}$ reflexión de los conceptos de paz, violencia, Cultura de Paz, del conflicto, así como un manejo eficaz y solución pacífica de sus conflictos. El programa de capacitación para los padres, denominado Familias con Cultura de Paz, buscó potencializar las habilidades de los padres y madres participantes para actuar como elementos de cambio de conciencia en sus hijos.

El programa consistió de seis sesiones, de una hora y media de duración, iniciando en marzo y concluyendo en junio del 2016. Durante las sesiones se trabajó con los padres de familia utilizando una metodología teórica y práctica, mediante la aplicación de dinámicas, exposición del contenido y de ejercicios de reflexión en grupo. Al finalizar cada sesión los participantes recibían copias de cuentos y actividades relacionadas con el tema expuesto para que lo compartieran con sus hijos.
La estructura de la capacitación se basó en dos programas internacionales similares, el programa Escuela de Familia (UNESCO, 2013), aplicado en Colombia en el periodo de 2009 a 2013 y el de Cultura de Paz en la Escuela (PREAL, 2006), realizado en Brasil durante el 2004 y 2005. Se revisaron los contenidos de ambos programas y se realizaron adaptaciones para la población de la región donde es aplicado.

Su contenido teórico consistió en la definición y reflexión de los elementos de la Cultura de Paz (ONU, 1999), particularmente del respeto, el diálogo, la tolerancia, la educación en el conflicto y la negociación como método de solución pacífica de conflictos. La capacitación en Cultura de Paz se presentó a los padres y madres como una plataforma de entrenamiento para que adquieran habilidades para ser entrenadores de paz para sus hijos.

La premisa del programa no era hacer sentir a los padres incompetentes en relación a la crianza de sus hijos, ya que esto sería una falta de respeto hacia ellos, además de que generaría resistencia y rechazo a participar; por el contrario el objetivo fue reconocer y empoderar su rol como formadores de sus hijos para proporcionarles, mediante la educación, la plataforma teórica y práctica para que ellos se capacitaran enseñando la Cultura de Paz a sus hijos, desarrollando en ellos conductas encaminadas a la convivencia en paz con sus pares y a la solución pacífica de conflictos. 
Al formar a los padres como capacitadores en Cultura de Paz se impacta en dos generaciones, los participantes y sus hijos. El conocimiento, reflexión y práctica de los valores, actitudes y comportamientos que conforman una cultura de paz, de acuerdo a la ONU (1999) generará cambios en los participantes:

- Recordarán valores que les fueron transmitidos a ellos mismos en su infancia, pero que por la influencia de la violencia cultural no llevaban a cabo en sus vidas y relaciones.

- Reconocerán la importancia de los valores, como el respeto y la tolerancia, para la educación en las interacciones humanas, individuales y sociales.

- Reforzarán la trascendencia de su rol como formadores de personas, de adultos en desarrollo, de individuos, de una nueva generación.

- Potencializarán su capacidad como formadores de sus hijos.

\section{Metodología}

Se utilizó un diseño de investigación preexperimental con padres de familia con niños en edad preescolar. Este diseño consiste primero en observar o medir un determinado grupo, segundo en introducir un factor que supuestamente producirá algún cambio en el mismo, y por último en volver a observar o medir el grupo luego de haber aplicado el factor, para ver si se registró el cambio esperado (Cazau, 2006). Por otro lado, Cea D’Ancona (2001), refiere que los diseños preexperimentales incluyen la modalidad de diseño pre test-post test de un único grupo, que es la forma de aplicación del cuestionario cuantitativo en la presente investigación.

El presente estudio se llevó a cabo en el municipio de San Nicolás de los Garza, ubicado en el Estado de Nuevo León, México. Para tener acceso a padres con niños en edad preescolar, se contactaron diversas instituciones educativas que ofrecen educación a niños en ese rango de edad. Se logró la aprobación por parte de la directora del Jardín de Niños Ezequiel Chávez, ubicado en la calle San Marcos, sin número, Colonia Villas de Santo Domingo. Se invitó a participar a los padres de los alumnos del jardín de niños, se presentaron 19 participantes, 17 mujeres y 2 hombres.

Para recolectar los datos se utilizó metodología cuantitativa y cualitativa. Se usó la encuesta como técnica de recolección cuantitativa por medio de la aplicación de un cuestionario de 26 ítems con la escala de respuesta Likert de acuerdo, siendo el mismo aplicado previo al curso y nuevamente al final del mismo, esto es lo que se conoce como pre y post test.

El tipo de muestreo seleccionado fue no probabilístico, también llamado muestreo no representativo, intencional, de criterio o determinístico. Debido a que este es considerado un estudio exploratorio que no sirve para hacer generalizaciones debido a que la muestra extraída puede no representar las características de toda la población (Rodríguez-Burgos, 
2012), sin embargo se hizo de esta manera debido al carácter y duración del programa en donde los involucrados solo pueden participar voluntariamente.

Para la comprobación cualitativa se utilizó el método de la entrevista semiestructurada. Se seleccionó debido a que permite conocer la experiencia vivida por la persona entrevistada y facilita el conocer su perspectiva, opiniones y percepción en relación al tema central de las preguntas del investigador. Así mismo, contar con una serie de preguntas permite explorar en la experiencia del entrevistado con un orden previamente establecido y facilita el desarrollo de confianza y el rapport necesarios para la investigación (Cea D’Ancona, 2001).

El estudio cualitativo, esto es, las entrevistas, se aplicaron a los mismos padres de familia que habían previamente participado en el programa Familias con Cultura de Paz, al concluir las seis sesiones del programa, esto se hizo con el fin de profundizar los cambios en la relación con sus hijos, por lo que decidieron participar en esta segunda parte cinco padres de familia, siendo cuatro mujeres y un hombre. En las cinco entrevistas semiestructuradas realizadas se respetó la ética del principio de confidencialidad ya que no se dan a conocer datos personales de los entrevistados, como nombres o particularidades.

\section{Resultados}

Con el objetivo de obtener información acerca de la tolerancia y el respeto se procedió a examinar los ítems del cuestionario cuantitativo que miden dichos valores. Así mismo, se realizó el análisis de las entrevistas semiestructuradas presentando los resultados relacionados con los valores que son el objeto de estudio del presente artículo.

\section{Respeto. Resultados cuantitativos}

Los ítems del cuestionario cuantitativo que miden el respeto son:

Me considero una persona con facilidad para expresar lo que pienso y siento.

Me siento satisfecho con la forma en que estoy educando a mis hijos/as.

Consulto con mis hijos/as sobre sus planes antes de planear mis actividades.

Me siento insatisfecho con la forma en que estoy educando a mis hijos/as.

Analizando los resultados de la prueba $t$ de Student, en relación al respeto, en la primera aplicación del cuestionario la media arrojó un valor de 3.3838, mayormente neutral o hacia la respuesta Ni de acuerdo ni en desacuerdo, pero con una leve tendencia hacia el desacuerdo. En comparación con la media de la aplicación post, 3.6938, hay un cambio con tendencia hacia lo positivo, lo cual indica un aumento en la manifestación del respeto en su relación con él mismo o ella misma, en la relación con sus hijos y con los demás. 


\section{Estadísticos de muestras relacionadas}

Tabla 1. Resultados $t$ de student para muestras relacionadas Respeto

\begin{tabular}{|l|c|c|c|c|}
\hline & Media & N & $\begin{array}{c}\text { Desviación } \\
\text { Tip. }\end{array}$ & $\begin{array}{c}\text { Error de } \\
\text { tip. de la } \\
\text { media }\end{array}$ \\
\hline $\begin{array}{l}\text { Respeto Pre test } \\
\text { Respeto Post test }\end{array}$ & 3,3838 & 13 &, 47912 &, 13288 \\
\hline
\end{tabular}

En el análisis de las frecuencias de los ítems del instrumento cuantitativo diseñados para evaluar el respeto el item Me considero una persona con facilidad para expresar lo que pienso y siento, se mide el respeto que tienen los encuestados a ellos mismos en relación a la expresión de sus pensamientos y sentimientos.

Me considero una persona con facilidad para expresar lo que pienso y siento. Pre y Post test

Tabla 2. Frecuencias, Pre y Post test

\begin{tabular}{|l|c|c|c|c|}
\hline & $\begin{array}{c}\text { Frecuencia } \\
\text { Pre test }\end{array}$ & $\begin{array}{c}\text { Porcentaje } \\
\text { Pre test }\end{array}$ & $\begin{array}{c}\text { Frecuencia } \\
\text { Post test }\end{array}$ & $\begin{array}{c}\text { Porcentaje } \\
\text { Post test }\end{array}$ \\
\hline En & 1 & 5,3 & 1 & 7,7 \\
desacuerdo & & & & \\
Ni de acuerdo & 5 & 26,3 & 5 & 23,1 \\
ni en & 9 & & 9 & \\
desacuerdo & & & & \\
De acuerdo & 4 & 47,4 & 4 & 23,1 \\
Totalmente & 19 & 21,1 & 19 & 46,2 \\
de acuerdo & & & & \\
\hline
\end{tabular}

Al comparar los resultados de la aplicación pre y post se encontró que, en la primera aplicación del cuestionario el 47,4\% de los encuestados respondieron estar de acuerdo en que se consideran personas con facilidad para expresar lo que piensan y sienten. El porcentaje de respuesta Totalmente de acuerdo, fue de $21,1 \%$. En comparación, en la aplicación posterior a la participación al programa Familias con Cultura de Paz, el porcentaje de respuesta más elevado fue Totalmente de acuerdo, subiendo de un $21,1 \%$ a un $46,2 \%$, con un incremento de 25,1 puntos porcentuales.

Esto indica un aumento en la confianza de los padres participantes en el programa, así como en la percepción de la capacidad de expresión. La manifestación de sentimientos y pensamientos es una demostración de autorespeto, además de promover el diálogo, lo que impacta en la disminución de la posibilidad de surgimiento del conflicto. Si se llega a detonar una situación conflictiva la facilidad de expresar pensamientos y emociones permite su resolución pacífica.

Me siento satisfecho con la forma en que estoy educando a mis hijos. Pre y Post test

Tabla 3. Frecuencias, Pre y Post test

\begin{tabular}{|l|c|c|c|c|}
\hline & $\begin{array}{c}\text { Frecuencia } \\
\text { Pre test }\end{array}$ & $\begin{array}{c}\text { Porcentaje } \\
\text { Pre test }\end{array}$ & $\begin{array}{c}\text { Frecuencia } \\
\text { Post test }\end{array}$ & $\begin{array}{c}\text { Porcentaje } \\
\text { Post test }\end{array}$ \\
\hline $\begin{array}{l}\text { En desacuerdo } \\
\text { Ni de acuerdo }\end{array}$ & 5 & 5,6 & & \\
ni en & 27,8 & 3 & 23,1 \\
desacuerdo & & & & \\
De acuerdo & 9 & 50,0 & 5 & 38,5 \\
Totalmente & 3 & 16,7 & 5 & 38,5 \\
de acuerdo & & & & \\
\hline
\end{tabular}

El item Me siento satisfecho con la forma en que estoy educando a mis hijos, en la aplicación pre test el $50 \%$ de los encuestados indicaron estar de acuerdo en sentirse satisfechos. En 
la aplicación post test se presenta un aumento del porcentaje de respuesta de Totalmente de acuerdo, aumentando de un $16,7 \%$ a un $38,5 \%$, subiendo un total de 21,8 puntos porcentuales. La satisfacción con la forma de crianza se relaciona con el autorrespeto, la aceptación y el reconocimiento de las habilidades parentales.

El conocimiento transmitido en el curso sobre los elementos de la Cultura de Paz y estrategias de negociación y solución pacífica de conflictos le proporcionan a los padres de familia herramientas adicionales, las que utilizaban para relacionarse y enseñar disciplina a sus hijos. Esto genera un aumento en la satisfacción respecto a la forma en la que educan a sus hijos, lo cual contribuye a mejorar la relación paterno filial, ya que los padres mejorarán su estado de ánimo. La satisfacción se relaciona estrechamente con la felicidad, si el padre de familia se siente feliz se relacionará de una forma positiva con sus hijos.

Consulto con mis hijos/as sobre sus planes antes de planear mis actividades. Pre y Post test

Tabla 4. Frecuencias, Pre y Post test

\begin{tabular}{|l|c|c|c|c|}
\hline & $\begin{array}{c}\text { Frecuencia } \\
\text { Pre test }\end{array}$ & $\begin{array}{c}\text { Porcentaje } \\
\text { Pre test }\end{array}$ & $\begin{array}{c}\text { Frecuencia } \\
\text { Post test }\end{array}$ & $\begin{array}{c}\text { Porcentaje } \\
\text { Post test }\end{array}$ \\
\hline $\begin{array}{l}\text { En desacuerdo } \\
\text { Ni de acuerdo }\end{array}$ & 5 & 5,3 & 1 & 8,3 \\
ni en & & 26,3 & 3 & 25,0 \\
desacuerdo & & & & \\
De acuerdo & 9 & 47,4 & 6 & 50,0 \\
Totalmente & 4 & 21,1 & 2 & 16,7 \\
de acuerdo & & & & \\
\hline
\end{tabular}

Respecto al item, en la primera aplicación del cuestionario el 47,4\% de los encuestados refirieron estar De acuerdo, indicando que consultan con sus hijos/as sobre sus planes antes de planear sus actividades. En la aplicación post test se observa que el $50 \%$ de los encuestados refirieron estar De acuerdo, presentando un aumento de 2,6 puntos porcentuales.

El considerar a los hijos antes de planear las actividades es una expresión de respeto hacia ellos. El conocimiento y reflexión acerca del potencial del aprendizaje por imitación en los niños y de la forma de enseñar respeto a los hijos mediante el ejemplo propicia el aumento de manifestaciones de este valor en las situaciones cotidianas. La comunicación al planear las actividades familiares ayuda a disminuir malos entendidos así como el surgimiento de conflictos, además facilita las interacciones entre los miembros.

Me siento insatisfecho con la forma en que estoy educando a mis hijos/as. Pre y Post test

Tabla 5. Frecuencias, Pre y Post test

\begin{tabular}{|l|c|c|c|c|}
\hline & $\begin{array}{c}\text { Frecuencia } \\
\text { Pre test }\end{array}$ & $\begin{array}{c}\text { Porcentaje } \\
\text { Pre test }\end{array}$ & $\begin{array}{c}\text { Frecuencia } \\
\text { Post test }\end{array}$ & $\begin{array}{c}\text { Porcentaje } \\
\text { Post test }\end{array}$ \\
\hline $\begin{array}{l}\text { Totalmente } \\
\text { en desacuerdo }\end{array}$ & 5 & 26,3 & 1 & 9,1 \\
En desacuerdo & 6 & 31,6 & 4 & 36,4 \\
Ni de acuerdo & 3 & 15,8 & 2 & 18,2 \\
ni en & & & & \\
desacuerdo & & 5,3 & 4 & 36,4 \\
De acuerdo & 1 & 21,1 & & \\
Totalmente & 4 & & & \\
de acuerdo & & & & \\
\hline
\end{tabular}

En relación al ítem, Me siento insatisfecho 
con la forma en que estoy educando a mis hijos/as, en la aplicación pre test destaca el porcentaje máximo de respuesta que el $31,6 \%$ de los encuestados están en desacuerdo en sentirse insatisfechos con la forma en la que están educando a sus hijos, lo que contrasta con un porcentaje de respuesta de Totalmente de acuerdo del $21,1 \%$, lo que puede indicar que la pregunta no fue entendida. En la aplicación post test el porcentaje de respuesta De acuerdo y En desacuerdo fueron iguales, de 36,4\%.

Debido a que los porcentajes de respuesta De acuerdo y En desacuerdo fueron similares se infiere que hubo confusión por parte de los participantes sobre lo que la pregunta quería medir. En posteriores evaluaciones se omitirá este item, ya que tenía la función de ser una pregunta de control, pero resultó confusa para los encuestados.

\section{Respeto. Resultados cualitativos}

El guión de la entrevista semi estructurada constó de 18 preguntas abiertas con la función de explorar la experiencia vivida por los padres participantes en el programa y a la aplicación práctica del contenido teórico expuesto. En relación al respeto se indagó acerca de la forma de relacionarse con ellos mismos y con sus hijos, sobre elementos de la relación paterno filial, como la comunicación y empatía hacia sus hijos.

\section{Respeto hacia en niño}

Tabla 6. Respeto hacia el niño

\begin{tabular}{|l|l|}
$\begin{array}{l}\text { Padres entrevistados que refirieron sentir más res- } \\
\text { peto hacia sus hijos }\end{array}$ & $44,74 \%$ \\
\hline
\end{tabular}

En los resultados cualitativos relacionados con el respeto se observó que el 44,74\% de los padres entrevistados refirieron que después de su participación en el programa Familias con Cultura de Paz sintieron más respeto hacia sus hijos. Manifestaron considerar más las necesidades del niño, sus limitaciones en cuestión de entendimiento y madurez. Una de las entrevistadas mencionó que antes trataba a su hijo con el mismo nivel de exigencia que un adulto y con las mismas expectativas de conducta, agregó que después de las sesiones comenzó a observar más a su hijo, ponerse en su posición y entenderlo un poco más.

Las relaciones entre padres e hijos, como el resto de las relaciones interpersonales, se componen de un lazo afectivo, un interés por el bienestar de la otra parte, confianza, respeto, comunicación. Estos elementos son las bases que permiten el inicio, desarrollo y permanencia de forma funcional de la relación interpersonal. La relación con sus padres será la primera relación interpersonal del niño, por lo cual es importante que esté fundamentada en el respeto.

El respeto es un valor que promueve que las interacciones se desarrollen de manera funcional, permite la comunicación y la convivencia pacífica. El respeto en las relaciones entre padres e hijos debe ser bilateral, el padre le corresponde enseñarlo mediante el ejemplo en el trato y las interacciones con el menor. La capacidad de conocer y respetar a los niños ayuda a clarificar y evitar conflictos relacionados con las expectativas de los padres. 


\section{Tolerancia. Resultados cuantitativos}

Los ítems del cuestionario cuantitativo que miden la tolerancia son:

Es fácil para mí aceptar que mi hijo/a tiene opiniones que son diferentes a las mías.

Me considero una persona que acepta fácilmente las opiniones diferentes a las mías.

Cuando las opiniones de los demás son diferentes a las mías pienso que están equivocados.

Cuando tengo mis actividades planeadas y algo me altera la planeación, acepto con facilidad los cambios.

En el análisis de los datos cuantitativos respecto a la tolerancia, los resultados de la prueba estadística $t$ de Student, realizada en el software SPSS, en la aplicación previa al programa Familias con Cultura de Paz la media fue de 3.5769, que es mayormente neutral o hacia la respuesta $\mathrm{Ni}$ de acuerdo ni en desacuerdo con tendencia hacia la respuesta De acuerdo. En comparación con la media de la aplicación post, 3.5577 , hay un cambio con leve tendencia hacia lo negativo, lo cual indica una disminución de la manifestación de la tolerancia respecto a opiniones, pensamientos y creencias diferentes a los propios, así como en su relación con él mismo o ella misma, con sus hijos y con los demás.

\section{Estadísticos de muestras relacionadas}

Tabla 7. Resultados $t$ de Student para muestras relacionadas Tolerancia

\begin{tabular}{|l|c|c|c|c|}
\hline & Media & $\mathbf{N}$ & $\begin{array}{c}\text { Desviación } \\
\text { Tip. }\end{array}$ & $\begin{array}{c}\text { Error de tip. } \\
\text { de la media }\end{array}$ \\
\hline $\begin{array}{l}\text { Tolerancia } \\
\text { Pre test } \\
\begin{array}{l}\text { Tolerancia } \\
\text { Post test }\end{array}\end{array}$ & 3,5769 & 13 &, 54376 &, 15081 \\
\hline
\end{tabular}

El análisis de las frecuencias de respuesta de la aplicación previa y la posterior a su participación en el programa Familias con Cultura de Paz de los ítems del cuestionario que miden la tolerancia arrojaron los siguientes resultados.

Es fácil para mi aceptar que mi hijo/a tiene opiniones que son diferentes a las mías. Pre y Post test

Tabla 8. Frecuencias, Pre y Post test

\begin{tabular}{|l|c|c|c|c|}
\hline & $\begin{array}{c}\text { Frecuencia } \\
\text { Pre test }\end{array}$ & $\begin{array}{c}\text { Porcentaje } \\
\text { Pre test }\end{array}$ & $\begin{array}{c}\text { Frecuencia } \\
\text { Post test }\end{array}$ & $\begin{array}{c}\text { Porcentaje } \\
\text { Post test }\end{array}$ \\
\hline $\begin{array}{l}\text { En desacuerdo } \\
\text { Ni de acuerdo }\end{array}$ & 2 & 10,5 & 1 & 8,3 \\
ni en & & & & 8,3 \\
desacuerdo & & 43,1 & 5 & 41,7 \\
De acuerdo & 8 & 47,4 & 5 & 41,7 \\
Totalmente & 9 & & & \\
de acuerdo & & & & \\
\hline
\end{tabular}

En el item Es fácil para mí aceptar que mi hijo/a tiene opiniones que son diferentes a las mías, en la primera aplicación del cuestionario el 47,4 \% de los encuestados respondieron estar Totalmente de acuerdo con que para ellos es fácil aceptar que su hijo/a tiene opiniones diferentes a las suyas. El porcentaje de respuesta De acuerdo fue de $42,1 \%$. En la aplicación poste- 
rior se observa una disminución leve en el porcentaje de la respuesta Totalmente de acuerdo, $41,7 \%$, con un decremento de 5,7 puntos porcentuales. En la respuesta De acuerdo el porcentaje de respuesta fue de $41,7 \%$, mostrando una leve disminución en el porcentaje.

En contraste, otra diferencia entre ambas aplicaciones es que la respuesta En desacuerdo no fue seleccionada por ningún participante en el pre test, mientras que en la post test el porcentaje de esta respuesta fue de 8,3. Así mismo, el porcentaje en la respuesta de acuerdo sigue siendo de más de $40 \%$. La aceptación de las opiniones de los hijos aunque sean diferentes a las propias es una manifestación de tolerancia. Que los padres consideren y acepten las opiniones de sus hijos desde la infancia promueve y facilita el proceso de toma de decisiones en los niños, además de que impacta benéficamente en su autoestima y confianza en ellos mismos.

Me considero una persona que acepta fácilmente las opiniones diferentes a las mías. Pre y Post Test

Tabla 9. Frecuencias, Pre y Post test

\begin{tabular}{|l|c|c|c|c|}
\hline & $\begin{array}{c}\text { Frecuencia } \\
\text { Pre test }\end{array}$ & $\begin{array}{c}\text { Porcentaje } \\
\text { Pre test }\end{array}$ & $\begin{array}{c}\text { Frecuencia } \\
\text { Post test }\end{array}$ & $\begin{array}{c}\text { Porcentaje } \\
\text { Post test }\end{array}$ \\
\hline $\begin{array}{l}\text { En desacuerdo } \\
\text { Ni de acuerdo }\end{array}$ & 1 & 5,3 & 1 & 7,7 \\
ni en & 4 & 21,1 & 3 & 23,1 \\
desacuerdo & & & & \\
$\begin{array}{l}\text { De acuerdo } \\
\text { Totalmente }\end{array}$ & 7 & 36,8 & 6 & 46,2 \\
de acuerdo & 7 & 36,8 & 3 & 23,1 \\
\hline
\end{tabular}

En el item Me considero una persona que acepta fácilmente las opiniones diferentes a las mías, en la aplicación pre se obtuvo el mismo porcentaje en las respuestas De acuerdo y Totalmente de acuerdo, $36,8 \%$, manifestando que los encuestados se consideran personas que aceptan fácilmente las opiniones de diferentes a las suyas. En la aplicación post se observa un aumento en el porcentaje de respuesta en De acuerdo, 46,2 \%, con un incremento de 9,4 puntos porcentuales, así mismo la respuesta Totalmente de acuerdo disminuyó en su porcentaje a un $23,1 \%$, con un decremento de 13,7 puntos porcentuales.

La aceptación de las opiniones de los demás es una expresión de la tolerancia en las relaciones interpersonales. La capacidad de aceptar opiniones distintas a las propias promueve el inicio y desarrollo del diálogo, posibilita incrementar y mejorar las relaciones personales con los demás. Así mismo la aceptación de opiniones puede disminuir la detonación de conflictos, impedir que se magnifiquen y permitir la búsqueda de soluciones que beneficien a ambas partes.

Cuando las opiniones de los demás son diferentes a las mías pienso que están equivocados. Pre y Post test

Tabla 10. Frecuencias Pre y Post test

\begin{tabular}{|l|c|c|c|c|}
\hline & $\begin{array}{c}\text { Frecuencia } \\
\text { Pre test }\end{array}$ & $\begin{array}{c}\text { Porcentaje } \\
\text { Pre test }\end{array}$ & $\begin{array}{c}\text { Frecuencia } \\
\text { Post test }\end{array}$ & $\begin{array}{c}\text { Porcentaje } \\
\text { Post test }\end{array}$ \\
\hline $\begin{array}{l}\text { Totalmente } \\
\text { en desacuerdo }\end{array}$ & 5 & 26,3 & 2 & 15,4 \\
En desacuerdo & 4 & 21,1 & 3 & 23,1 \\
$\begin{array}{l}\text { Ni de acuerdo } \\
\text { ni en }\end{array}$ & 8 & 42,1 & 6 & 46,2 \\
desacuerdo & & & & \\
$\begin{array}{l}\text { De acuerdo } \\
\text { Totalmente } \\
\text { de acuerdo }\end{array}$ & 1 & 5,3 & 1 & 7,7 \\
& 1 & 5,3 & 1 & 7,7 \\
\hline
\end{tabular}


En el item Cuando las opiniones de los demás son diferentes a las mías pienso que están equivocados, en la primera aplicación del cuestionario hay una postura neutral en relación a la afirmación de si cuando las opiniones de los demás son diferentes a la de los encuestados piensan que están equivocados. El 42,1 \% respondió estar Ni de acuerdo ni en desacuerdo, mientras que el 21,1 \% eligió la respuesta En desacuerdo. En la aplicación post continuó la postura neutral, con un $46,2 \%$ de respuesta $\mathrm{Ni}$ de acuerdo ni en desacuerdo, con un leve cambio hacia lo positivo ya que el porcentaje de respuesta En desacuerdo fue de 23,1\%, aumentando 2 puntos porcentuales. El respeto a las opiniones de los demás es una expresión de tolerancia.

La tolerancia es fundamental para el diálogo y la convivencia pacífica. La capacidad de considerar opiniones distintas a las propias sin juzgarlas como equivocadas permite la escucha activa y la curiosidad sobre la experiencia del otro y el fundamento de su pensamiento. Así mismo, en la relación entre padres e hijos el no juzgar como erróneas las opiniones distintas de los niños permite a los adultos explorarlas con apertura, lo que genera oportunidades de diálogo y convivencia, dando como resultado un beneficio para su relación interpersonal.

Cuando tengo mis actividades planeadas y algo me altera la planeación, acepto con facilidad los cambios. Pre y Post test
Tabla 11. Frecuencias, Pre y Post test

\begin{tabular}{|l|c|c|c|c|}
\hline & $\begin{array}{c}\text { Frecuencia } \\
\text { Pre test }\end{array}$ & $\begin{array}{c}\text { Porcentaje } \\
\text { Pre test }\end{array}$ & $\begin{array}{c}\text { Frecuencia } \\
\text { Post test }\end{array}$ & $\begin{array}{c}\text { Porcentaje } \\
\text { Post test }\end{array}$ \\
\hline En desacuerdo & 2 & 10,5 & & \\
Ni de acuerdo & 9 & 47,4 & 41,7 & 5 \\
ni en & & & & \\
desacuerdo & & 21,1 & 41,7 & 5 \\
De acuerdo & 4 & 21,1 & 16,7 & 2 \\
Totalmente & 4 & & & \\
de acuerdo & & &
\end{tabular}

En el item Cuando tengo mis actividades planeadas y algo me altera la planeación, acepto con facilidad los cambios en la aplicación previa a las sesiones del programa, el 47,4\% de los encuestados refirieron estar Ni de acuerdo ni en desacuerdo con la afirmación de que cuando tienen sus actividades planeadas y algo altera la planeación aceptan con facilidad los cambios. En la aplicación post se observa un aumento en el porcentaje de la respuesta De acuerdo, de un $21,1 \%$ en la medición previa se incrementa a $41,7 \%, 20$ puntos porcentuales. La aceptación de los cambios es una manifestación de la tolerancia.

Las actitudes y reacciones de los padres ante los cambios son fácilmente transmitidos a los niños mediante el aprendizaje por imitación. El nivel de tolerancia a la frustración que manifiestan los padres será observado y posteriormente aprendido por sus hijos, este comportamiento se interpreta como lo que se permite hacer cuando el menor se encuentre en situaciones similares. Si bien el desarrollo de la tolerancia a la frustración es gradual, se relaciona con la madurez y requiere de la exposición progresiva a situaciones o elementos 
frustrantes, es positivo para los niños observar que sus padres pueden manejar su frustración de formas constructivas.

\section{Tolerancia. Resultados cualitativos}

A continuación se presentan los resultados cualitativos relacionados con la tolerancia. En la entrevista semiestructurada se le cuestionó a los padres sobre su nivel de tolerancia antes y después de participar en el curso, se arrojaron los siguientes datos.

\section{Nivel de Tolerancia antes del curso}

Tabla 12. Nivel de tolerancia antes del curso

\begin{tabular}{|l|c|}
\hline Poco tolerante & $40 \%$ \\
\hline Demasiado tolerante & $20 \%$ \\
\hline Muy tolerante & $20 \%$ \\
\hline Tolerante & $20 \%$ \\
\hline
\end{tabular}

Respecto al nivel de tolerancia antes de participar en el programa de Familias con Cultura de Paz, el 40 \% refirieron ser poco tolerantes. Una de las entrevistadas refirió que no era tolerante con su hijo debido a que llegaba cansada de su trabajo y quería descansar, agregó que los recursos que utilizaba para comunicarse con su hijo eran los gritos. Un bajo nivel de tolerancia puede propiciar el uso de métodos agresivos o violentos para moldear la conducta de los niños (UNICEF México, 2014).

El $20 \%$ respondieron que antes de participar en el programa eran demasiado tolerantes, explicando que no ponían límites a sus hijos, les facilitaban algunas cosas al grado en que les impedían vivir experiencias que podían generarles aprendizajes. Una de las encuestadas refirió debido al carácter explosivo de su hijo le restringía el contacto con otros niños, afirmó que después del programa se dio cuenta que en lugar de beneficiar a su hijo le afectaba negativamente, ya que lo privaba de experiencias que podían ayudarle a tolerar su frustración. Los encuestados mencionaron que eran demasiado tolerantes por falta de herramientas diferentes a los gritos y los golpes para establecer límites con sus hijos.

El $20 \%$ de los entrevistados afirmó ser muy tolerante al momento de iniciar el programa, así mismo otro $20 \%$ de los participantes refirió ser tolerante. Ninguno de los entrevistados mencionó no ser tolerante antes de su participación en las sesiones.

\section{Cambios en el nivel de tolerancia}

Tabla 13. Cambios en el nivel de tolerancia antes del curso

\begin{tabular}{|l|l|}
\hline Más tolerante & $33,3 \%$ \\
\hline Grito menos & $29,6 \%$ \\
\hline Más paciencia & $25,9 \%$ \\
\hline Me desespero menos & $11,1 \%$ \\
\hline
\end{tabular}

En relación a los cambios en el nivel de tolerancia después de haber participado en el programa Familias con Cultura de Paz, el 33,33 \% de los padres entrevistados afirmó ser más tolerante. Al respecto, Stiffelman (2015) refiere que una comunicación funcional y adecuada entre padres e hijos requiere de madurez, consciencia y responsabilidad por parte de los pa- 
dres, ya que es necesario reconocer y respetar las opiniones de la otra persona, respetar sus sentimientos y tener tolerancia y consideración durante el diálogo.

Además del cambio en el nivel de tolerancia los resultados de las entrevistas semiestructuradas muestran un aumento en la comunicación, en el uso del diálogo y un aumento en la atención que se le da al niño y en el tiempo de convivencia. La presencia de la tolerancia permite y facilita los encuentros entre padres e hijos, así mismo impactan positivamente en la eficacia de la relación padre e hijo y en la confianza en las habilidades de crianza, que es una categoría del autorreconocimiento de las habilidades parentales.

El 29,63 \% de los entrevistados refirió usar menos los gritos como forma de comunicación con sus hijos. La educación en las herramientas y conceptos de la disciplina positiva genera un cambio en la forma de comunicación y relación entre padres e hijos, ya que el uso de métodos violentos para disciplinar a los niños se sostiene culturalmente debido a la ignorancia, la confusión y la violencia cultural. UNICEF México (2014) refiere que el concepto de disciplina es en ocasiones confundido o mal interpretado como una excusa para aplicar o utilizar métodos agresivos de modificación de conducta, como por ejemplo gritos, golpes, insultos o humillaciones. La línea entre la disciplina y el castigo como forma de violencia física es fina y es importante delimitarla mediante la educación, la definición y diferenciación de ambas.
El 25,93 \% de los entrevistados respondieron que despues de su participación en el programa percibieron que tenían más paciencia. Educar y reflexionar sobre los elementos de la Cultura de Paz genera en los padres mayor conciencia de la importancia de formar a sus hijos con respeto y usando estrategias positivas. Esto impacta positivamente en el nivel de paciencia y tolerancia de los padres hacia sus hijos, lo que beneficia la relación paterno filial y la autoconfianza de los padres.

La subcategoría anterior se relaciona con el siguiente resultado, el 11,11\% de los entrevistados refirieron desesperarse menos con sus hijos. El aumento en el nivel de tolerancia se manifiesta en un incremento en la paciencia al respecto de la relación con los niños. Con mayor paciencia el padre se desespera menos, puede utilizar estrategias para solucionar conflictos y manejar la conducta no deseada del niño; asi mismo puede relacionarse con él de forma más relajada.

\section{Conclusiones}

En los resultados acerca del respeto ambos métodos, cualitativo y cuantitativo, coinciden en que los participantes del programa declaran un aumento en las manifestaciones de respeto hacia ellos mismos y en sus relaciones con los demás. Puede concluirse que dicho aumento es producto de la participación del programa Familias con Cultura de Paz debido a que durante el mismo se les recordó y reforzó la importancia del respeto en las relaciones intra e interpersonales y que los participantes se sin- 
tieron respetados a lo largo de las sesiones. Puede observarse que se logró un cambio de comportamiento en los participantes del programa, sin embargo se requiere de seguimiento para explorar si existe cambio en sus actitudes o creencias.

En relación a las diferencias de resultados obtenidos respecto la tolerancia, se encontró evidencia de que el método con el cual se obtuvieron los datos impacta en los resultados obtenidos. Samaniego (2010) menciona que para recolectar la información acerca de qué hechos o cualidades le atribuyen los padres a la conducta de sus hijos y que a su vez impacta en su nivel de tolerancia, se pueden utilizar dos métodos, las preguntas abiertas o cerradas.

En la misma publicación, Samaniego (2010) cuestiona si el uso de uno u otro método impactan en la respuesta de los padres. En el presente estudio se utilizaron ambos, preguntas abiertas en el guión cualitativo y preguntas cerradas en el cuestionario cuantitativo, y se encontraron diferencias significativas en las respuestas.

$\mathrm{Al}$ respecto es posible interpretar que la disparidad entre los resultados cuantitativos y cualitativos puede explicarse en relación al método utilizado para recolectar los datos considerando los siguientes factores:

1. Es probable que alguno de los ítems del cuestionario cuantitativo no fue entendido correctamente.

2. Las preguntas abiertas dan pie a la interpretación subjetiva del investigador.
3. En la entrevista semiestructurada el entrevistado puede responder lo que piensa que el entrevistador quiere escuchar.

Otro de los factores a considerar para explicar la disminución en el nivel de tolerancia que se presentó en los resultados cuantitativos se manifiesta en uno de los resultados de la entrevista semiestructurada. Al cuestionar a los entrevistados acerca de su nivel de tolerancia antes de participar en el programa el $20 \%$ respondieron que antes de participar en el programa eran demasiado tolerantes, explicando que no ponían límites a sus hijos, les facilitaban algunas cosas al grado en que les impedían vivir experiencias que podían generarles aprendizajes. Los encuestados mencionaron que eran demasiado tolerantes por falta de herramientas diferentes a los gritos y los golpes para establecer límites con sus hijos.

Considerando esta respuesta, se puede deducir que la disminución del nivel de tolerancia expresado en los resultados cuantitativos se debe a que los padres participantes adquirieron herramientas diferentes a los gritos y golpes con las cuales les fue posible poner límites a la conducta de sus hijos de forma constructiva, dando como resultado una disminución de la excesiva tolerancia que manifestaron en la entrevista. Con este enfoque, se interpreta como positivo el hecho de que el nivel de tolerancia haya disminuido ya que poner límites a los niños es necesario para su formación.

Un área de oportunidad del estudio fue la 
ausencia de un grupo control para descartar que los cambios observados hayan sido producidos por estímulos no considerados en la investigación. Así mismo, en posteriores aplicaciones del cuestionario serán eliminados los ítems que generaron confusión en los encuestados. En futuras investigaciones se realizará un seguimiento a los participantes del programa para conocer su experiencia y explorar la continuidad del uso de las herramientas transmitidas durante el curso.

\section{Referencias}

Bajo, I., Campillo, H., Pérez, G., Gener, B., Gallego, J. \& Padrino, A. (2011). Agresividad infantil. Chimbote, Perú: Editorial Universidad César Vallejo.

Cárdenas-Flores, V., Cosiatado-Carrasco, G. \& Livia-Vicente, S. (2011). Contenidos televisivos violentos asociados a la conducta agresiva de niños de 8 a 12 años. Revista de Enfermería Herediana, 4(2), 49-55.

Cazau, P. (2006). Introducción a la Investigación en Ciencias Sociales ( $3^{\mathrm{a}} \mathrm{Ed}$.). Buenos Aires, Argentina.

Cea D’Ancona, M. (2001). Metodología cuantitativa. Estrategias y técnicas de Investigación Social (Sintesis Sociológica ed.). Madrid, España.

Cornella i Canals, J. \& Llusent i Guillament, A. (2010). Agresividad y violencia en el niño y en el adolescente. Programa "Salud i Escola”. Girona, España.

Flores Millán, L. (2011). Análisis de una Problemática Educativa: Conducta agresiva en niños de preescolar. México, México.

Modelo de Desarrollo Económico de Navarra (2010). Escuelas de Familia MODERNA Bloque III Documentación de las Competencias. Escuelas de Familia MODERNA.

Muñiz Muriel, C. (noviembre de 2012). Curso sobre Análisis Bivariados y Multivariados con SPSS. Monterrey, Nuevo León, México: Universidad Autónoma de Nuevo León.

ONU (1999). Resolución A/53/243.

Pérez, G.J. (2009). La relación entre ética y comunicación en sí mismo como otro respecto a las categorías de verdad, libertad y justicia. Acta Fenomenológica Latinoamericana, III, 723-741.

PREAL (2006). Cultura de Paz en las escuelas. Santiago, Chile: Editorial San Marino.

Rodríguez-Burgos, K. (2012). Investigación cuantitativa: Diseño, técnicas, muestreo y análisis cuantitativo. En K.A. Sáenz López, F.J. Gorjón Gómez, M. Gonzalo Quiroga \& C.M. Díaz Barrado, Metodología para investigaciones de alto impacto en las Ciencias Sociales y Jurídicas. Madrid, España.

Samaniego, V.C. (2010). Escala de tolerancia parental hacia los comportamientos infantiles, elaboración y validación. Revista Latinoamericana de Psicología, 42(2), 203-214.

Stiffelman, S. (2015). Parenting with presence ( $1^{\text {a }}$ Ed.). Novato, California, Estados Unidos: New World Library.

UNESCO (1995). Declaración de Principios so- 
bre la Tolerancia. París: UNESCO.

UNESCO (2013). Sistematización de Experiencias en Escuelas de América Latina y el Caribe. Educación para la Paz, Convivencia Democrática y Derechos Humanos. Organización de las Naciones Unidas para la Educación, la Ciencia y la
Cultura. Santiago: UNESCO.

UNICEF México (2014). Violencia y maltrato. Recuperado el 27 de octubre de 2014, de UNICEF México: http://www.unicef. org/mexico/spanish/proteccion_6932. htm

von Hildebrand, D. (2004). La importancia del respeto en la educación. Educación y Educadores, (7), 221-228. 